\title{
Long Non-Coding RNA Expression Profiles for the Characterization of Different Bladder Cancer Grade
}

\author{
Yue-Peng Cao Jun Zhou ${ }^{\mathrm{a}}$ Wei-Jian Li ${ }^{\mathrm{a}}$ Yang Shao ${ }^{\mathrm{c}}$ Shu-Yun Zheng ${ }^{\mathrm{b}}$ \\ Tian Tian $^{d}$ Kai-peng Xie ${ }^{c, e} \quad$ Xiang Yan ${ }^{\text {a,f }}$
}

aDepartment of Urology, Drum Tower Hospital, Medical School of Nanjing University, Institute of Urology, Nanjing University, Nanjing, 'bepartment of Critical Care Medicine, Jiangsu Cancer Hospital, Jiangsu Institute of Cancer Research, Nanjing Medical University Affiliated Cancer Hospital, Nanjing, 'Nanjing Maternity and Child Health Care Institute, The Affiliated Obstetrics and Gynaecology Hospital of Nanjing Medical University, Nanjing Maternity and Child Health Care Hospital, Nanjing, dDepartment of Child Health Care, the First Affiliated Hospital of Nanjing Medical University, Nanjing, eDepartment of Women Health Care, The Affiliated Obstetrics and Gynaecology Hospital of Nanjing Medical University, Nanjing Maternity and Child Health Care Hospital, Nanjing, China, 'Department of Urology, Anqing Petrochemical Hospital, Nanjing Gulou Hospital Group, Anqing, China

\section{Key Words}

IncRNAs • Bladder cancer • Expression profile • Progression

\begin{abstract}
Background/Aims: Bladder cancer (BC) is one of the most frequent urologic tumors worldwide. However, long non-coding RNA(IncRNA) expression profiles in BC progression remain unclear. This study aimed to explore IncRNA expression profiles in different grades of bladder cancer and normal urothelium tissues. Methods: We performed high-throughput sequencing in BC tissues of different grade and obtained the expression profiles of its IncRNAs. Then, aberrantly expressed IncRNAs were validated by quantitative reverse transcription polymerase chain reaction (RT-PCR). Gene Ontology (GO) and pathway analyses were used to investigate the potential function of these IncRNAs. Co-expresson network was constructed to explore the relationship between IncRNAs and target mRNAs. Results: We identified 252 aberrantly expressed IncRNAs in high-grade BC while compared to low-grade BC, and 269 IncRNAs in high-grade $B C$ while compared to normal urothelium. Notably, we found 33 overlapped IncRNAs. Subsequently, 7 IncRNAs were selected from the overlapped part and confirmed by RT-PCR. GO and pathway analyses showed that these dysregulated IncRNAs participated in cell migration, cell adhesion, as well as Ras signaling pathway. Co-expression network and The Cancer Genome Atlas (TCGA) data showed LUCAT1 and CCNB1 had positive relationship in regulating the progress of bladder cancer. Conclusion: Our findings revealed the significant role of IncRNAs in the development process of bladder cancer.

Y. Cao and J. Zhou contributed equally to this work. Published by S. Karger AG, Basel

\footnotetext{
Xiang Yan

and Kai-peng Xie

Department of Urology, Drum Tower Hospital, Medical School of Nanjing University, Institute of Urology,

Nanjing University, Nanjing; Department of Urology, Anqing Petrochemical Hospital,

Nanjing Gulou Hospital Group, Anqing (China); E-Mail yanxiang@nju.edu.cn; kaipengxie@njmu.edu.cn
} 


\section{Cellular Physiology Cell Physiol Biochem 2018;50:1154-1163 \begin{tabular}{l|l|l} 
DOl: 10.1159/000494542 & $\begin{array}{l}\text { O 2018 The Author(s). Published by S. Karger AG, Basel } \\
\text { www.karger.com/cpb }\end{array}$ \\
\hline
\end{tabular}}

Cao et al.: IncRNA Expression Profiles of Different Bladder Cancer Grade

\section{Introduction}

Bladder cancer (BC) is the ninth most frequently-diagnosed cancer worldwide, and ranks 13th in terms of deaths [1]. In China, bladder cancer is the most common urologic neoplasms with 80, 500 new cases diagnosed and 32, 900 deaths in 2015 [2]. The majority histologic type of bladder cancer is urothelial carcinoma, $75 \%$ of which are non-muscle invasive bladder cancer (NMIBC) and others are muscle-invasive bladder cancer (MIBC) [3]. The recurrence rate of NMIBC within 5 years range from $50 \%$ to $70 \%$, and about 17 $45 \%$ of NMIBC cases were found to develop [4]. Currently, high-grade invasive bladder cancer doesn't have an ideal treatment, available approaches include radical cystectomy and chemotherapy [5]. Both of them remarkably impact patient's quality of life and survival time [6], therefore, it is still imperative to explore the underlying potential mechanisms of malignant progression of bladder cancer.

Long non-coding RNAs (IncRNAs) are transcripts more than200 nucleotides in length and would not be translated into proteins [7]. More than $80 \%$ of the mammalian genome is composed of IncRNAs [8]. Accumulating evidence support that IncRNAs are involved in the malignant process of cancer, such as tumorigenesis and metastasis [9-12]. During the past years, several dysregulation IncRNAs have been discovered and found to play important roles in bladder cancer. For examples, IncRNA-UNMIBC was up-regulated in NMIBC tissues compared with normal tissues and the expression level was significantly associated with recurrence rate [13]. Analyzing clinical specimens and data from The Cancer Genome Atlas (TCGA), Mónica et al. found lncRNA HOTAIR expression increased in recurrent and highgraded tumors and was associated with poor prognosis [14]. Moreover, lncRNA could also function as a cancer-suppressor gene. He et al. proved MIR31HG expression was negatively associated with TNM stage. However, there is very limited information on IncRNAs expression which differs in BC progress from low grade BC to high grade BC.

In this study, we performed high-throughput sequencing to explore the expression of IncRNAs and mRNAs in high-grade BC tissues while compared to low-grade BC as well as compared to tissues from normal controls. We hope our finding may help to discover potential biomarkers and therapeutic targets for bladder cancer.

\section{Materials and Methods}

\section{Patients and tissue specimens}

This study was approved by the Ethics Committee of Nanjing Drum Tower Hospital, The Affiliated Hospital of Nanjing University Medical School. Bladder cancer was diagnosed by histopathology examination. Written Informed consent were signed by patients before collecting tissue samples. In total, 13 low-grade BC tissues obtained from patients who underwent TURBT, and 13 high-grade BC tissues and matched adjacent normal tissues were collected from patients who underwent radical cystectomy at the Nanjing Drum Tower Hospital between 2013 and 2016. All tissue samples were added to tubes with RNAlater and snapfrozen in liquid nitrogen immediately and stored at $-80^{\circ} \mathrm{C} .3$ low grade cancer tissues, 3 high grade cancer tissues and 3 normal urothelial tissues were randomly selected for high-throughput sequencing analysis.

\section{RNA extraction}

According to the manufacturer's protocol, total RNA was isolated from samples by using TRIzol reagent (Invitrogen, Carlsbad, CA, USA). The purity and quantity of total RNA was evaluated using a Nano Drop nD1000 spectrophotometer (OD $260 \mathrm{~nm}$, Nano Drop, Wilmington, De, USA). Then, RNA integrity was assessed with standard denaturing agarose gel electrophoresis.

RNA-sequencing and data preprocessing

The quantity and purity of the total RNA were measured using an ultra-micro spectrophotometer (OD $260 \mathrm{~nm}$, Nano Drop, Wilmington, De, USA). Then, the lncRNA sequencing libraries were constructed according to the manufacture instruction. The libraries were conducted to sequencing by using IlluminaHiSeq 4000 


\section{Cellular Physiology Cell Physiol Biochem 2018;50:1154-1163 \begin{tabular}{l|l|l} 
and Biochemistry Published onlIne: 24 October 2018 & $\begin{array}{l}\text { (c) } 2018 \text { The Author(s). Published by S. Karger AG, Basel } \\
\text { www.karger.com/cpb }\end{array}$ \\
\hline
\end{tabular} \\ Cao et al.: IncRNA Expression Profiles of Different Bladder Cancer Grade}

sequencing (Illumina, Inc., San Diego, CA, USA). The raw data obtained from RNA-sequencing was checked by FastQC (http://www.bioinformatics.bbsrc.ac.uk/projects/fastqc). Date filtering include removing adapters, contamination reads and low-quality reads. Then, the clean reads of IncRNAs and mRNAs were mapped to the human reference genome (release hg19) by applying the HISAT2 program (V2-2.0.1). Stringtie (v1.3.0) and fragments per kilobase of exon per million fragments mapped (FPKM) was used to identify the significantly differentially expressed genes with cut-off criteria: $P<0.05$ and $\mid \log 2$ fold change| $>1$.

\section{Quantitative real-time PCR}

Total RNA (1 $\mu \mathrm{g}$ ) was reverse-transcribed to cDNA by the PrimeScript RT reagent kit (Takara, Japan). The IncRNA expression levels were measured by RT-PCR with an ABI VII7 PCR System (Applied Biosystems, USA) using SYBR Green PCR assays (Takara, Japan), according to the manufacturer's guidelines.

The reactions were incubated in a 384 -well optical plate at $95^{\circ} \mathrm{C}$ for $10 \mathrm{~min}$ for initial denaturation, followed by $40 \mathrm{PCR}$ cycles of incubation at $95^{\circ} \mathrm{C}$ for $15 \mathrm{~s}, 60^{\circ} \mathrm{C}$ for $30 \mathrm{~s}$, and then $72^{\circ} \mathrm{C}$ for 30 s. Melting curve analysis was used to confirm the specificity of PCR product. Relative expression levels were determined using the comparative CT method $\left(2^{-\Delta \Delta \mathrm{CT}}\right.$ method). The expression levels of lncRNAs were normalized to the endogenous reference gene GAPDH, and each sample was run in triplicate for analysis.

\section{Functional group analysis}

GO and KEGG analyses were applied to identify the biological functions of the aberrantly expressed lncRNAs. The IncRNA data were imported into the Database for Annotation, Visualization and Integrated Discovery (DAVID) (david.abcc.ncifcrf.gov/tools.jsp). GO analysis was applied to analyze the main function of the differential expression genes according to the Gene ontology project. KEGG enrichment analysis was used to analyze the biological pathways, involving the differentially expressed lncRNAs. A P value below 0.05 was considered to indicate significance.

\section{PPI network construction}

Search Tool for the Retrieval of Interacting Genes (STRING) database (https://string-db.org/) records the experimental and predicted interaction information between proteins by calculating their combined score [15]. In our study, common differential expression genes in high-grade BC vs. low-grade BC, and highgrade BC vs. normal, with the combined score of PPIs greater than 0.7 were screened out. Thus, PPI networks were constructed based on the information of STRING database. In particular, the selection of combined score was based on the magnitude of observed PPIs. Moreover, the PPI network was visualized using Cytoscape software [16]. Additionally, the degree of nodes (proteins) in the PPI network was calculated, indicating the number of interacting partners. The proteins with higher node degree in each PPI network were considered as hubs.

\section{IncRNA-mRNA co-expression network}

The IncRNA-mRNA co-expression network was constructed to determine their relationship among these differentially expressed IncRNAs and mRNAs. The lncRNAs and mRNAs that had Pearson correlation coefficients equal to or greater than 0.99 were selected, and from those a network was constructed from the groups using the Cytoscape program (http://www.cytoscape.org).

\section{Results}

\section{LncRNAs expression profile in different grade bladder cancer and Normal urothelium}

We performed high-throughput sequencing in 3 normal bladder tissues, 3 low-grade BC tissues and 3 high-grade BC tissues. $P<0.05$ and $\mid \log 2$ fold change $\mid>1$ were selected as the cutoff. Compared with normal tissues, the high-grade BC tissues expressed 269 lncRNAs consisted of 172 up-regulated and 97 down-regulated lncRNAs. Compared to low-grade BC tissues, the high-grade BC tissues expressed 252 lncRNAs consisted of 172 up-regulated and 80 down-regulated lncRNAs. Heatmaps were used to assess those variations in lncRNA expression (Fig. 1A\&B). With further analysis, we found 33 lncRNAs were overlapped and had the same direction of expression change in the above two groups (Table 1). We also found 


\section{Cellular Physiology Cell Physiol Biochem 2018;50:1154-1163

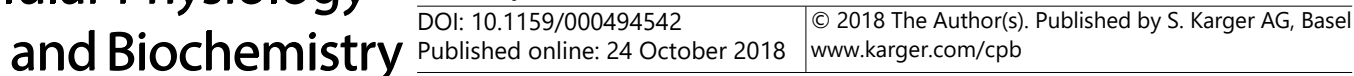 \\ Cao et al.: IncRNA Expression Profiles of Different Bladder Cancer Grade}

465 lncRNAs expressed differently in low-grade BC tissues compared to normal tissues, but these genes don't have gradient differences with high-grade BC and low-grade BC (Fig. S1 for all supplemental material see www.karger.com/10.1159/000494542/).

mRNAs expression profile in different grade bladder cancer and Normal urothelium

In the meanwhile, we identified 1688 mRNAs (1307 up-regulated and 381 downregulated) in high-grade BC vs low-grade BC tissues, and 1215 mRNAs (554 up-regulated and 661 down-regulated) in high-grade BC vs normal tissues (Table S1\&S2). Similarly, 268 mRNAs were overlapped in two groups (Table S3).

RT-PCR validation of overlapped IncRNAs in different grade bladder cancer and normal urothelium tissues.

To validate high-throughput sequencing results of dysregulated IncRNAs, we collected 10 normal urothelium tissues, 10 low-grade BC and 10 high-grade BC samples. We choose IncRNAs according to the following rules. First, combined with the fold change of lncRNAs expression in two groups. Second, the same direction of expression change in two groups.

A

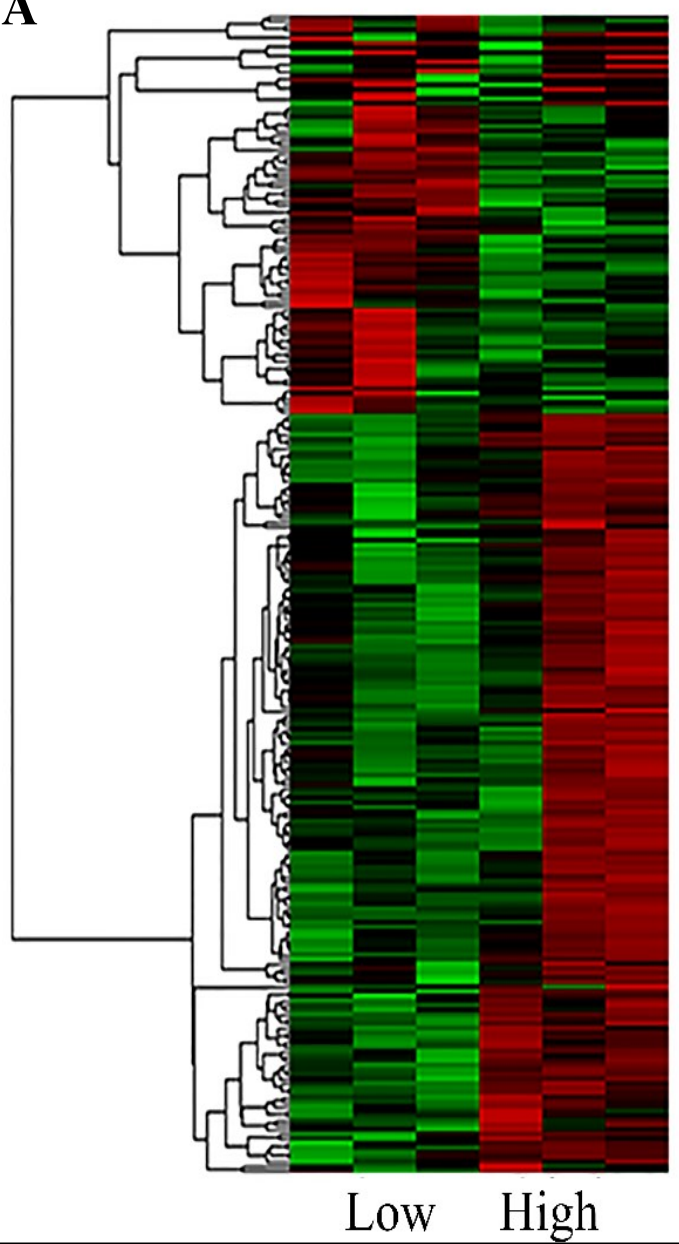

B

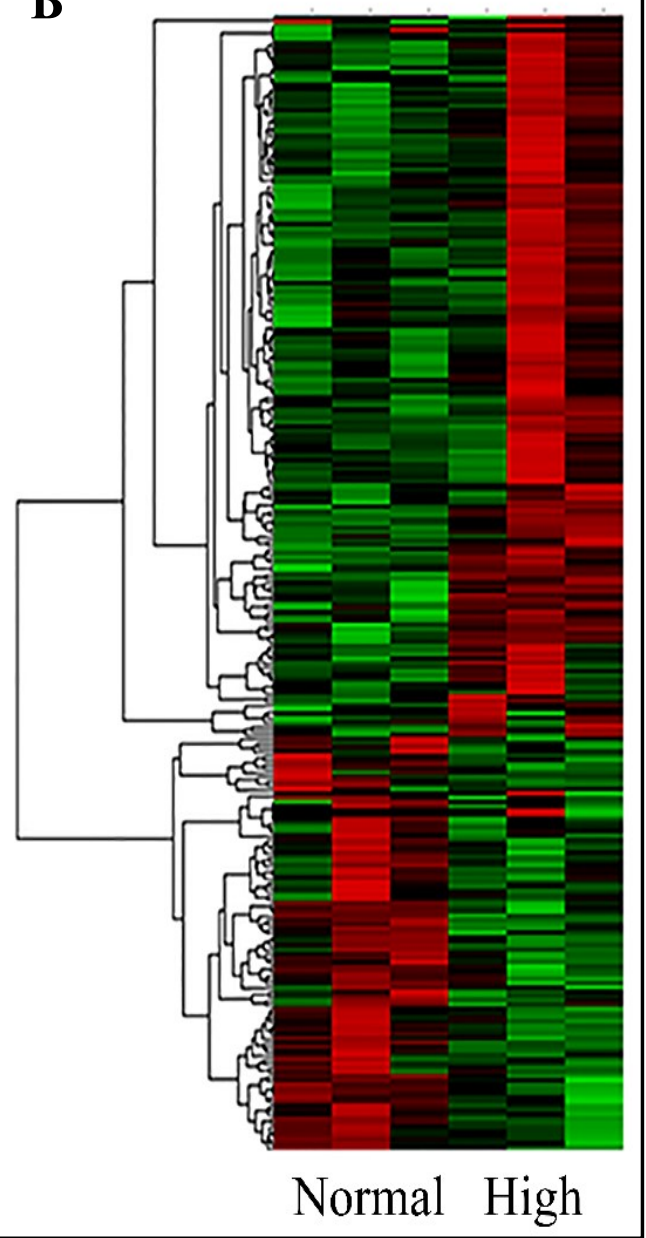

Fig. 1. Differentially expressed lncRNAs in different grade bladder cancer and normal urothelium tissues. (A) Heatmap of expression profiles for the 269 lncRNAs that showed significant expression changes (172 up-regulated and 97 down-regulated), green through red color indicates low to high expression level. (B) Heatmap of expression profiles for 252 lncRNAs that showed significant expression changes (172 upregulated and 80 down -regulated), green through red color indicates low to high expression level. 


\section{Cellular Physiology Cell Physiol Biochem 2018;50:1154-1163

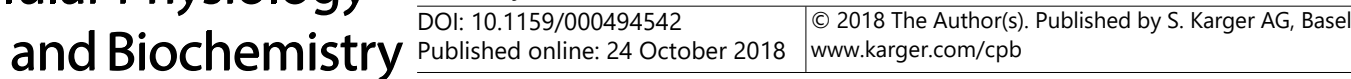 \\ Cao et al.: IncRNA Expression Profiles of Different Bladder Cancer Grade}

Third, the information of gene status which was validated in UCSC database. Finally, we selected 7 of the 33 lncRNAs for RT-PCR quantification. The expression of IncRNA AC114812.8, LINP1, LUCAT1 were significantly increased, whereas the expression of lincRNA LINC00886, AP001046.5, CTC-55802.1, LINC01011 were markedly decreased (Fig. 2A-G). The RTPCR results were consistent with the expression trends of the highthroughput sequencing data.

GO analysis and pathway analysis of the differentially expressed IncRNAs.

Studies have proved that IncRNAs could exert its biological function by regulating the neighboring coding genes. Thus, we used GO analysis and pathway analysis to assess the biological significance of lncRNAs through partner mRNAs. Gene ontology analysis and pathway analysis were performed on significantly dysregulated lncRNAs in the two groups. We identified the top 10 enriched GOs in high-grade BC compared with low-grade $\mathrm{BC}$, including cell differentiation, GTPase mediated signal transduction and cell spreading and so on, (Fig. 3A). The top 10 enriched GOs in high-grade BC compared with normal urothelium, including cell differentiation, positive regulation of GTPase activity, cell migration and cell adhesion and so on (Fig. 3B).

Pathway analysis revealed 10 pathways in each subgroup. Compared with high-grade $\mathrm{BC}$ and low-grade BC, the MAPK signaling pathway, pathways in cancer and Ras signaling pathway were significantly enriched (Fig. 3C). Ras signaling pathway, cGMP-PKG signaling pathway and vascular smooth muscle contraction were significantly enhanced in high-grade $\mathrm{BC}$ with normal urothelium (Fig. 3D).

\section{PPI network analysis of overlapped mRNAs}

Based on the information of the STRING database, the PPI networks were constructed by $268 m R N A s$, which were overlapped of high-grade BC with low-grade BC and high-grade BC with normal urothelium groups. The PPI network analysis included 55 nodes and 69 edges. The highest connectivity degrees were CCNB1 (cyclin B1, degree = 13), RAD51 (RAD51 recombinase, degree $=7$ ) and IL8 (interleukin 8, degree $=6$ ) (Fig. S2).

\section{Co-expression of IncRNAs and $m R N A s$}

We also constructed IncRNA-mRNA co-expression networks in 33 lncRNAs and 268mRNAs in two groups. The co-expression network was composed of 89 nodes and 
Cellular Physiology Cell Physiol Biochem 2018;50:1154-1163

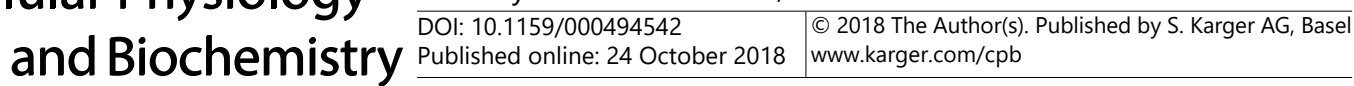

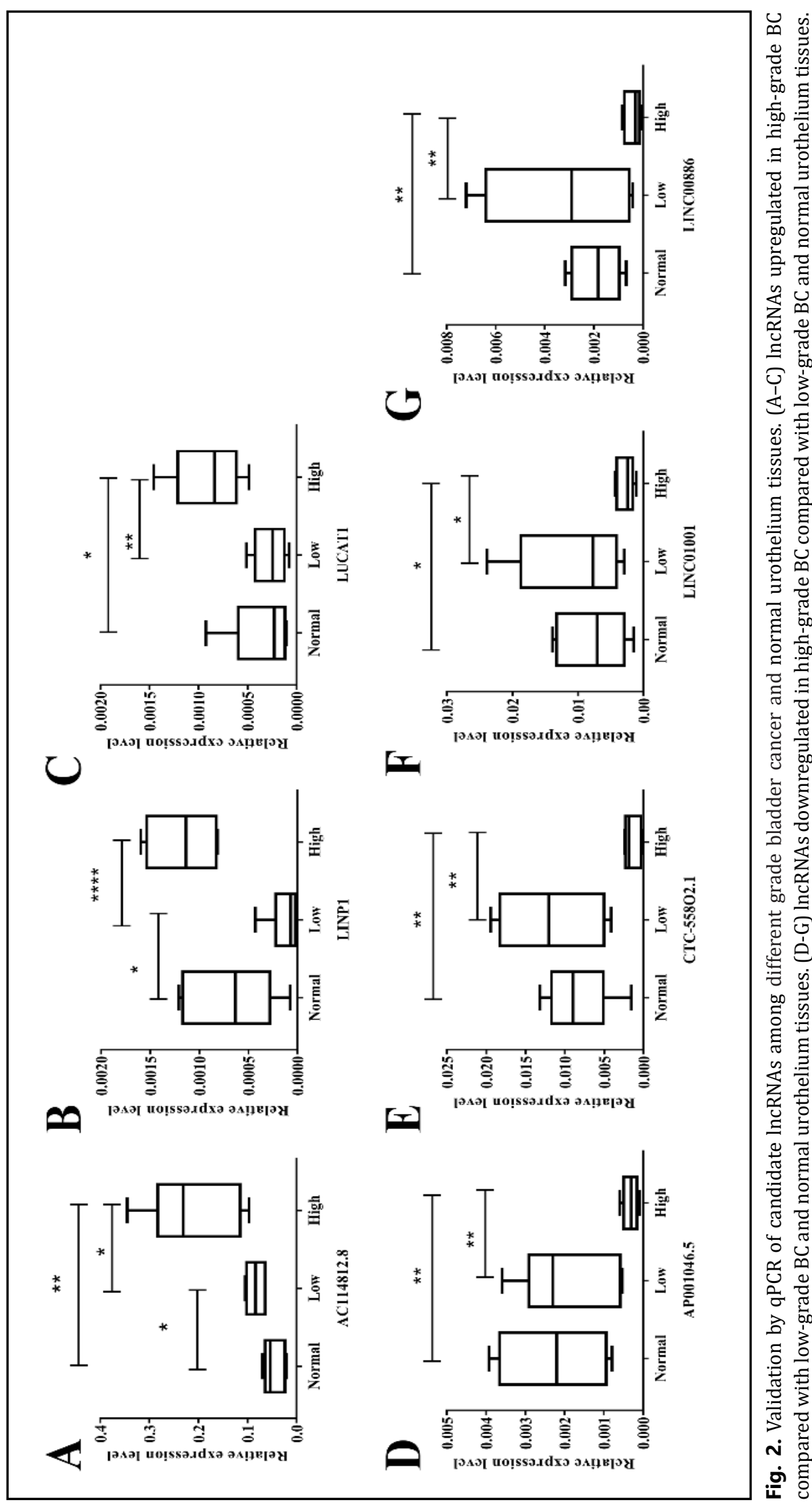




\section{Cellular Physiology Cell Physiol Biochem 2018;50:1154-1163 \begin{tabular}{ll|l} 
and Biochemistry Published online: 24 October 2018 & $\begin{array}{l}\text { (c) } 2018 \text { The Author(s). Published by S. Karger AG, Basel } \\
\text { www.karger.com/cpb }\end{array}$
\end{tabular}

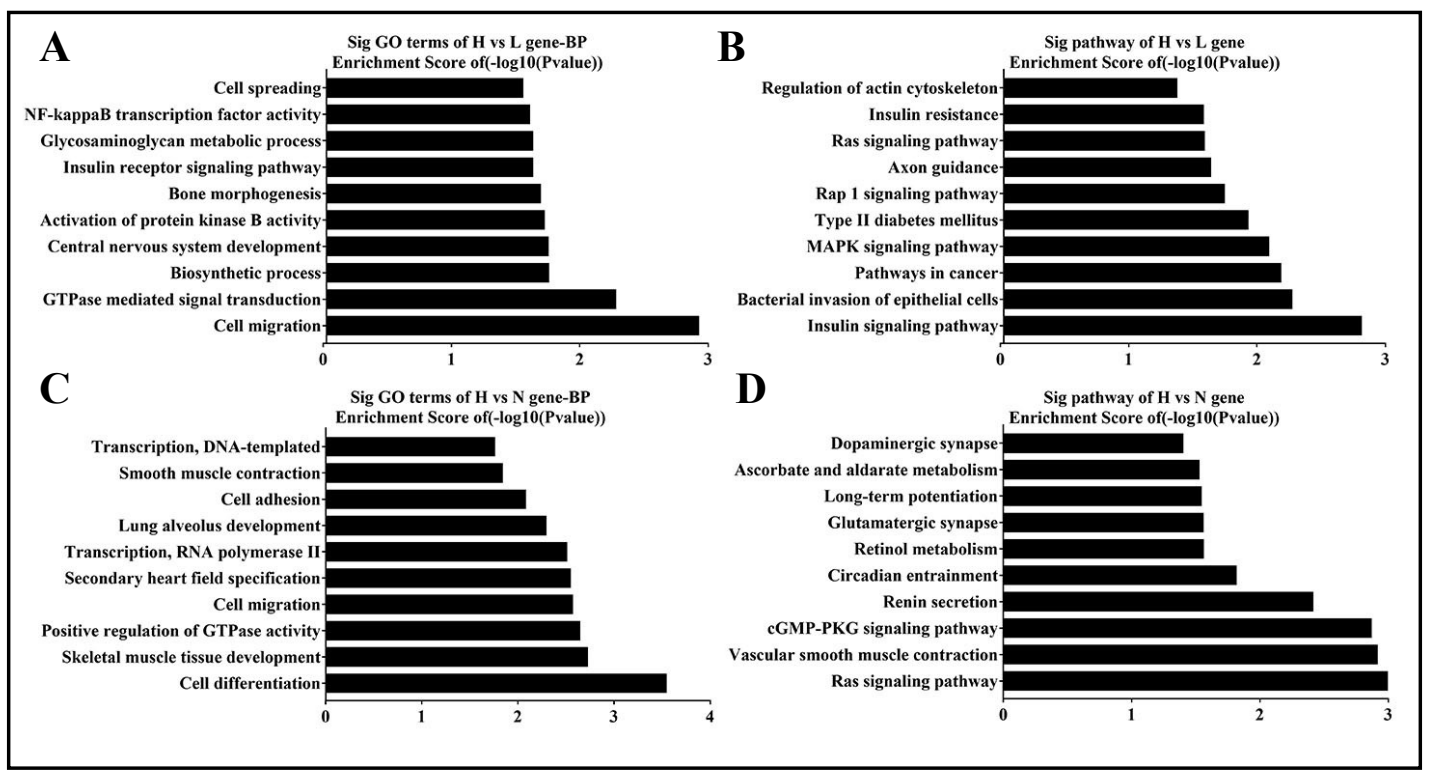

Fig. 3. GO analysis and KEGG analysis of the differentially expressed lncRNAs. (A-B)Top ten GO terms of biological processes and pathways for IncRNAs between high-grade BC and low-grade BC. (C-D) Top ten GO terms of biological processes and pathways corresponding to the lncRNAs between high-grade BC and normal urothelium tissues.

124 edges between 10 lncRNAs and 79 coding genes in high-grade BC with low-grade BC group (Fig. S3A). Among them, LncRNA01011, LUCAT1 and XLOC_096449 were significantly enriched. The co-expression network constructed of high-grade $\mathrm{BC}$ and normal urothelium included 139 nodes and 228 edges (Fig. S3B). The highest enriched genes were LncRNA01011, LncRNA00886 and XLOC_235536. In consistent with our research, The Cancer Genome Atlas (TCGA) Bladder urothelial carcinoma database (http://ibl.mdanderson.org/tanric/design/ basic/index.html) proved that LUCAT1 was expressed differently in high-grade BC vs lowgrade $\mathrm{BC}$ and had statistical significance in the diagnosis of papillary vs non-papillary bladder cancer (Fig. S4). What's more, TCGA database also showed that LUCT1 has a connection with CCNB1 and the correlation score was $0.527\left(P<3.4 \times 10^{\wedge-8}\right)($ Fig. S4).

\section{Discussion}

In recent years, mounting studies have demonstrated that lncRNAs take part in cancer molecular biology, such as tumorigenesis, invasion and transition, while there is still little information between the pathological grades progress and lncRNAs expression in bladder cancer. Therefore, we performed high-throughput sequencing to detect IncRNAs levels in bladder cancer tissues of different grade and normal tissues.

We identified 269 and 252 dysregulated lncRNAs respectively in two groups and 33 lncRNAs which were not only differentially expressed between high-grade BC and low-grade $\mathrm{BC}$ but also altered between high-grade BC and normal urothelium. Among them, it has been reported that low expression of MEG3 was associated with poor recurrence-free survival in bladder cancer [17]. The decreased of BDNF-AS expression is correlated with patients' advanced clinical stage and associated with shorter overall survival in retinoblastoma [18]. We further validated 7 lncRNAs (AC114812.8, LINP1, LUCAT1, LINC00886, AP001046.5, CTC-55802.1, LINC01011) in other independent tissues. Our results were consistent with the expression trends of the sequencing data. LINP1 significantly overexpressed in basal breast cancer and regulated repair of DNA breaks [19]. LINC01011 expressed differentially in intrahepatic cholangiocarcinoma and adjacent normal tissues [20]. CTC-55802.1 


\section{Cellular Physiology Cell Physiol Biochem 2018;50:1154-1163 \begin{tabular}{l|l} 
DOI: 10.1159/000494542 & O) 2018 The Author(s). Published by S. Karger AG, Basel \\
www.karger.com/cpb
\end{tabular} \\ Cao et al.: IncRNA Expression Profiles of Different Bladder Cancer Grade}

participated in the mTOR pathways network of gonadotrophin adenomas. Notably, one lncRNA has a variety of biological functions in the tumor and aroused our attention. LUCAT1, also named as SCAL1, located on chromosome 5, was first Characterized by Philip Thai et al. in 2013 [21]. It was reported that LUCAT1 was critical for the progression of glioma and regulated proliferation and invasion of glioma cells through miR-375 [22]. Clinical samples analysis showed that higher expression of LUCAT1 was associated with tumor size, tumornode-metastasis (TNM) stage and overall survival (OS). Besides, LUCAT1 also showed to modulate drug resistance $[23,24]$.

To explore the potential molecular mechanisms of these IncRNA, we used GO and pathway analyses. The GO analysis revealed that 'cell adhesion', 'GTPase activity' appeared in two groups. Pathway analysis also indicated MAPK signaling pathway, pathways in cancer and Ras signaling pathway were significantly enriched. It is supposed that these dysregulated IncRNAs in different grade bladder cancer and normal urothelium may participate in biological processes of cancer [25-28]. Bryan RT1 et al. found that N- and P-cadherin related to the cadherin cell adhesion and was the potential targets for the inhibition of cancer progression in bladder cancer [29]. Tomasetti et al. proved DNA replication errors mutations were responsible for two-thirds of the mutations in human cancers by analyzing cancer genome sequencing and epidemiological data [30]. It has also been proved that Foxo3 circular RNA could retard cell cycle progression by binding to CDK2 and p21 [31]. These biological functions may be associated with bladder cancer progression.

We constructed a protein-protein interaction (PPI) network based on the overlap of dysregulated mRNAs in two groups. CCNB1 gathered the highest connectivity of nodes. CCNB1 inhibition induced colorectal cancer apoptotic death and proliferation in vitro and tumor growth in vivo [32]. Huang $L$ et al. found that PBRM1 could block the G2/M transition by repressing cyclin B1, and PBRM1 was associated with advanced tumor stage, low differentiation grade and worse patient outcome [33]. While little demonstrate CCNB1 play a role in different grade bladder cancer. so, we speculate that CCNB1 may participate in the development or progression of bladder cancer. Consistent with our hypothesis, gene expression profiling identified that CCNB1 signature was associated with disease recurrence, stage and grade in 102 patients with NMIBC. What's more, gene network analyses and experimental assays showed that FOXM1-CCNB1-Fanconi anemia pathways might mediated NMIBC recurrence. In our study, IL8 is the second highest enriched connectivity nodes. Surprising, further investigation indicates that IL-8-stimulated cell proliferation correlates with alteration of cell cycle distribution by increasing levels of cell cycle-regulated Cyclin D1 and Cyclin B1 proteins as well as activation of PI3K/Akt and Raf/MEK/ERK [34]. Therefore, IL8 and CCNB1 may have potential function in the progress of bladder cancer.

To discern the potential connections between the overlapped lncRNAs and mRNAs, we built a lncRNA and mRNA co-expression network. LUCAT1 got a higher clustering coefficient with CCNB1 both in two groups. Other studies indicated that LUCAT1and CCNB1 play important roles in the biological processes, such as cell cycle, DNA damage response and cell apoptosis [35-37]. At the same time, TRANRIC web site also indicates there are some connections between LUCAT1 and CCNB1. Therefore, we speculate LUCAT1 and CCNB1 may participate in the progress of different grade bladder cancer.

\section{Conclusion}

In conclusion, we explored the aberrantly expressed lncRNAs and mRNAs in different grade cancer by high throughput sequencing. Our results suggested that those dysregulated genes including LUCAT1, LINC01011, LINC00886, CCNB1, IL8 might synergistically contribute to the progress of bladder cancer based on the bioinformatic analysis and RT-PCR. There are, however, some limitations in our study. Firstly, the specific molecular mechanism about the progress of bladder cancer remain unknown. Secondly, the biological roles of those novel dysregulated IncRNAs, such as LINC01011, LINC00886 and XLOC-235536, and how they 


\section{Cellular Physiology Cell Physiol Biochem 2018;50:1154-1163 \begin{tabular}{ll|l} 
and BiOChemistry & DOl: 10.1159/000494542 & $\begin{array}{l}\text { Published online: 24 October 2018 The Author(s). Published by S. Karger AG, Basel } \\
\text { www.karger.com/cpb }\end{array}$
\end{tabular} \\ Cao et al.: IncRNA Expression Profiles of Different Bladder Cancer Grade}

were involved in bladder cancer were not investigated. Our study provides potential lncRNA targets and direction for further investigations on the progress of bladder cancer.

\section{Acknowledgements}

This research was supported by the National Natural Science Fund of China (Nos. 81772712, 81702569), the Natural Science Foundation of Jiangsu Province (BK20170151).

\section{Disclosure Statement}

All authors state that they have no conflicts of interest.

\section{References}

1 Siegel RL, Miller KD, Jemal A: Cancer Statistics, 2017. CA Cancer J Clin, 2015;65:5-29.

2 Chen W, Zheng R, Baade PD, Zhang S, Zeng H, Bray F, Jemal A, Yu XQ He J: Cancer statistics in China, 2015. CA Cancer J Clin, 2016;66:115-132.

-3 Kamat AM, Hahn NM, Efstathiou JA, Lerner SP, Malmström PU, Choi W, Guo CC, Lotan Y, Kassouf W: Bladder cancer. Lancet 2016;388:2796.

4 Chang SS, Boorjian SA, Chou R, Clark PE, Daneshmand S, Konety BR, Pruthi R, Quale DZ, Ritch CR, Seigne JD: Diagnosis and Treatment of Non-Muscle Invasive Bladder Cancer: AUA/SUO Guideline. J Urol 2016;196:1021-1029.

5 Alfred WJ, Lebret T, Compérat EM, Cowan NC, De SM, Bruins HM, Hernández V, Espinós EL, Dunn J, Rouanne M: Updated 2016 EAU Guidelines on Muscle-invasive and Metastatic Bladder Cancer. Eur Urol 2017;71:462-475.

6 Antoni S, Ferlay J, Soerjomataram I, Znaor A, Jemal A, Bray F: Bladder Cancer Incidence and Mortality: A Global Overview and Recent Trends. Eur Urol, 2017;71:96.

7 Iyer MK, Niknafs YS, Malik R, Singhal U, Sahu A, Hosono Y, Barrette TR, Prensner JR, Evans JR, Zhao S: The landscape of long noncoding RNAs in the human transcriptome. Nat Genet 2015;47:199-208.

8 Deniz E, Erman B: Long noncoding RNA (lincRNA), a new paradigm in gene expression control. Deniz E, Erman B. Long noncoding RNA (lincRNA), a new paradigm in gene expression control [J]. Funct Integr Genomics, 2017, 17(2-3):135-143.

-9 Xu TP, Wang YF, Xiong WL, Ma P, Wang WY, Chen WM, Huang MD, Xia R, Wang R, Zhang EB: E2F1 induces TINCR transcriptional activity and accelerates gastric cancer progression via activation of TINCR/STAU1/ CDKN2B signaling axis. Cell Death Dis 2017;8:e2837.

10 Xiong Y, Wang L, Li Y, Chen M, He W, Qi L: The Long Non-Coding RNA XIST Interacted with MiR-124 to Modulate Bladder Cancer Growth, Invasion and Migration by Targeting Androgen Receptor (AR). Cell Physiol Biochem 2017;43:405.

11 Wang Y, Wang Y, Li J, Zhang Y, Yin H, Han B: CRNDE, a long-noncoding RNA, promotes glioma cell growth and invasion through mTOR signaling. Cancer Lett 2015;367:122-128.

12 Chen Y, Peng Y, Xu Z, Ge B, Xiang X, Zhang T, Gao L, Shi H, Wang C, Huang J: LncROR Promotes Bladder Cancer Cell Proliferation, Migration, and Epithelial-Mesenchymal Transition. Cell Physiol Biochem 2017;41:2399-2410.

13 Zhang S, Zhong G, He W, Yu H, Huang J, Lin T: IncRNA Up-Regulated in Nonmuscle Invasive Bladder Cancer Facilitates Tumor Growth and Acts as a Negative Prognostic Factor of Recurrence. J Urol 2016;196:12701278.

14 Martínezfernández M, Feber A, Dueñas M, Segovia C, Rubio C, Fernandez M, Villacampa F, Duarte J, Lópezcalderón FF, Ma JG: Analysis of the Polycomb-related lncRNAs HOTAIR and ANRIL in bladder cancer. Clin Epigenetics 2015;7:109.

15 Szklarczyk D, Morris JH, Cook H, Kuhn M, Wyder S, Simonovic M, Santos A, Doncheva NT, Roth A, Bork P: The STRING database in 2017: quality-controlled protein-protein association networks, made broadly accessible. Nucleic Acids Res 2017;45:D362-D368. 


\section{Cellular Physiology Cell Physiol Biochem 2018;50:1154-1163 \begin{tabular}{l|l|l} 
and Biochemistry Published online: 24 October 2018 & $\begin{array}{l}\text { ○ } 2018 \text { The Author(s). Published by S. Karger AG, Basel } \\
\text { www.karger.com/cpb }\end{array}$ \\
\hline
\end{tabular}}

16 Smoot ME, Ono K, Ruscheinski J, Wang PL, Ideker T: Cytoscape 2.8: new features for data integration and network visualization. Bioinformatics 2011;27:431-432.

17 Duan W, Du L, Jiang X, Wang R, Yan S, Xie Y, Yan K, Wang Q, Wang L, Zhang X: Identification of a serum circulating lncRNA panel for the diagnosis and recurrence prediction of bladder cancer. Oncotarget 2016;7:78850-78858.

18 Shang W, Yang Y, Zhang J, Wu Q: Long noncoding RNA BDNF-AS is a potential biomarker and regulates cancer development in human retinoblastoma. Biochem Biophys Res Commun 2018;497:1142-1148.

19 Zhang Y, He Q, Hu Z, Feng Y, Fan L, Tang Z, Yuan J, Shan W, Li C, Hu X: Long noncoding RNA LINP1 regulates repair of DNA double-strand breaks in triple-negative breast cancer. Nat Struct Mol Biol 2016;23:522.

20 Yang W, Yuan L, Xia S, Xu J, Xie J: Genome-wide analysis of long noncoding RNA and mRNA co-expression profile in intrahepatic cholangiocarcinoma tissue by RNA sequencing. Oncotarget 2017;8:26591-26599.

-21 Thai P, Statt S, Chen CH, Liang E, Campbell C, Wu R: Characterization of a Novel Long Noncoding RNA, SCAL1, Induced by Cigarette Smoke and Elevated in Lung Cancer Cell Lines. Am J Respir Cell Mol Biol 2013;49:204.

22 Gao YS, Liu XZ, Zhang YG, Liu XJ, Li LZ: Knockdown of long non-coding RNA LUCAT1 inhibits cell viability and invasion by regulating miR-375 in glioma. Oncol Res 2018;26:307-318.

23 Han Z, Shi L: Long non-coding RNA LUCAT1 modulates methotrexate resistance in osteosarcoma via miR200c/ABCB1 axis. Biochem Biophys Res Commun 2017;495:947-953.

-24 Zheng ZG, Hong X, Suo SS, Xu XL, Ni MW, Gu LH, Wei C, Wang LY, Ye Z, Bing T: The Essential Role of H19 Contributing to Cisplatin Resistance by Regulating Glutathione Metabolism in High-Grade Serous Ovarian Cancer. Sci Rep 2016;6:26093.

25 Eleveld TF, Oldridge DA, Bernard V, Koster J, Daage LC, Diskin SJ, Schild L, Bentahar NB, Bellini A, Chicard M: Relapsed neuroblastomas show frequent RAS-MAPK pathway mutations. Nat Genet 2015;47:864-871.

26 Burotto M, Chiou VL, Lee JM, Kohn EC: The MAPK pathway across different malignancies: a new perspective. Cancer 2015;120:3446-3456.

27 Wu XS, Wang XA, Wu WG, Hu YP, Li ML, Ding Q, Weng H, Shu YJ, Liu TY, Jiang L: MALAT1 promotes the proliferation and metastasis of gallbladder cancer cells by activating the ERK/MAPK pathway. Cancer Biol Ther 2014;15:806-814.

28 Li HJ, Sun XM, Li ZK, Yin QW, Pang H, Pan JJ, Li X, Chen W: LncRNA UCA1 Promotes Mitochondrial Function of Bladder Cancer via the MiR-195/ARL2 Signaling Pathway. Cell Physiol Biochem 2017;43:2548.

29 Bryan RT: Cell adhesion and urothelial bladder cancer: the role of cadherin switching and related phenomena. Philos Trans R Soc Lond B Biol Sci. 2015;370:20140042.

-30 Tomasetti C, Li L, Vogelstein B: Stem cell divisions, somatic mutations, cancer etiology, and cancer prevention. Science 2017;355:1330-1334.

-31 Du WW, Yang W, Liu E, Yang Z, Preet D, Yang BB: Foxo3 circular RNA retards cell cycle progression via forming ternary complexes with p21 and CDK2. Nucleic Acids Res 2016;44:2846.

32 Fang Y, Hong Y, Xiao L, Xu J, Cai X: Chk1-induced CCNB1 overexpression promotes cell proliferation and tumor growth in human colorectal cancer. Cancer Biol Ther 2014;15:1268-1279.

-33 Li H, Yang P, Zhong G, Xie W, Wen D, Bo W, Xu C, Peng G, Wang H, Wu S: PBRM1 suppresses bladder cancer by cyclin B1 induced cell cycle arrest. Oncotarget 2015;6:16366-16378.

-34 Kim SK, Roh YG, Park K, Kang TH, Kim WJ, Lee JS, Leem SH, Chu IS: Expression Signature Defined by FOXM1-CCNB1 Activation Predicts Disease Recurrence in Non-Muscle-Invasive Bladder Cancer. Clin Cancer Res 2014;20:3233-3243.

35 Ou Y, Ma L, Ma L, Huang Z, Zhou W, Zhao C, Zhang B, Song Y, Yu C, Zhan Q: Overexpression of cyclin B1 antagonizes chemotherapeutic-induced apoptosis through PTEN/Akt pathway in human esophageal squamous cell carcinoma cells. Cancer Biol Ther 2013;14:45-55.

-36 Zimmermann M, Arachchige-Don AS, Donaldson MS, Dallapiazza RF, Cowan CE, Horne MC: Elevated cyclin G2 expression intersects with DNA damage checkpoint signaling and is required for a potent G2/M checkpoint arrest response to doxorubicin. J Biol Chem 2012;287:22838-22853.

37 Lohberger B, Leithner A, Stuendl N, Kaltenegger H, Kullich W, Steineckerfrohnwieser B: Diacerein retards cell growth of chondrosarcoma cells at the G2/M cell cycle checkpoint via cyclin B1/CDK1 and CDK2 downregulation. BMC Cancer 2015;15:891. 